\section{Predictors of mortality in Middle East respiratory syndrome (MERS)}

\begin{abstract}
We evaluated the clinical characteristics, cytokine/chemokine concentrations, viral shedding and antibody kinetics in 30 patients with Middle East respiratory syndrome (MERS), including 6 nonsurvivors admitted to 3 MERS-designated hospitals. Old age, low albumin, altered mentality and high pneumonia severity index score at admission were risk factors for mortality. In addition, severe signs of inflammation at initial presentation (at hospital days 1-4), such as high inducible protein-10 $(p=0.0013)$, monocyte chemoattractant protein-1 $(p=0.0007)$ and interleukin $6(p=0.0007)$ concentrations, and poor viral control (high viral load at hospital days 5-10, $p<0.001)$ without adequate antibody titres (low antibody titre at hospital days $11-16, p=0.07$ ) during the course of disease, were associated with mortality.
\end{abstract}

\section{INTRODUCTION}

The mechanisms by which Middle East respiratory syndrome coronavirus (MERS-CoV) causes severe illness and fatal outcomes in humans are largely unknown. Progressive immune-associated injury, hypercytokinaemia, and inadequate adaptive immune responses have been suggested as possible mechanisms. However, there are limited data on immune responses in MERS-infected patients. ${ }^{12}$ The combined interpretation of clinical manifestations with cytokine/chemokine concentrations, viral loads and immune responses has helped in understanding the pathogenesis of many viral infections. We therefore analysed the clinical manifestations together with detailed cytokine/chemokine concentrations, viral shedding and antibody kinetics in patients with MERS. We also attempted to determine clinical, serological and immunological factors for fatal outcomes of patients with MERS.

\section{METHODS}

All MERS-confirmed patients who were admitted to three MERS-designated hospitals between 20 May 2015 and 18 July 2015 were prospectively enrolled. MERS-CoV infection was confirmed by positive real-time
Key messages

What is the key question?

What are the pathophysiological mechanisms underlying mortality in Middle East respiratory syndrome (MERS)?

What is the bottom line? Severe signs of inflammation at initial presentation, such as high inducible protein-10, monocyte chemoattractant protein-1 and interleukin-6 concentrations, and poor viral control without adequate antibody titres during the course of disease, are strongly associated with mortality in MERS-infected patients.

\section{Why read on?}

This study is the first to investigate the clinical, serological and immunological factors for fatal outcomes of patients with MERS, which are important for understanding the mechanisms by which MERS-coronavirus causes severe illness/fatal outcomes in humans and for developing the prognostic markers for survival of patients.

RT-PCR at the Korea Centre for Diseases Control and Prevention. We stored leftover specimens, including blood samples and respiratory specimens. About $500 \mu \mathrm{L}$ of plasma samples were stored at $-70^{\circ} \mathrm{C}$ for viral load analysis, and the remaining plasma was irradiated with 6 million rad from a ${ }^{60} \mathrm{Co}$ gamma source for cytokine and antibody analysis, as described previously. ${ }^{3}$ Respiratory specimens (sputum or tracheal aspirates) were obtained similarly and stored at $-70^{\circ} \mathrm{C}$. The institutional review boards agreed that informed consent should be waived in accordance with the hospital policies for emergent responses. The detailed procedures for MERS-CoV RT-PCR, cytokine analysis, and MERS-CoV serology are described in the online technical appendix. For statistical analyses, categorical variables were compared with Fisher's exact test or the $\chi 2$ test, as appropriate, and continuous variables were compared with the Mann-Whitney U test or Student t test. Cytokine/chemokine concentrations were compared using repeated measures analysis of variance or the MannWhitney U test. All tests of significance were two-tailed and $p$ values of $\leq 0.05$ were considered significant.

\section{RESULTS}

A total of 30 patients with MERS confirmed by MERS-CoV-specific RT-PCR of respiratory specimens were enrolled in this study. Their baseline clinical characteristics and outcomes are shown in table 1 . Old age, low albumin, altered mentality and high pneumonia severity index (PSI) score at admission were identified as risk factors for mortality. However, initial WBC count, platelet count, liver enzyme, and C-reactive protein were not associated with mortality.

Detailed expression profiles of cytokines/chemokines in response to MERS-CoV infection during hospitalisation are shown in figure 1 and supplementary figure 1 . Serum concentrations of inducible protein-10 (IP-10), monocyte chemoattractant protein-1 (MCP-1) and interleukin-6 (IL-6) during hospitalisation were significantly higher in the patients who died than in those who recovered (figure 1A-C), and, more importantly, these cytokines/chemokines concentrations during the early stage of MERS (HD 1-4) were particularly elevated in non-survivors compared with survivors (figure $1 \mathrm{D}-\mathrm{F}, \mathrm{p}=0.0013$ for IP-10, $p=0.0007$ for MCP-1 and $p=0.0007$ for IL-6).

The median time (IQR) between taking a sample which showed a negative result with RT-PCR for MERS-CoV and symptom onset was 19 days (15-21). Detailed kinetics of viral loads in respiratory specimens of survivors and non-survivors are shown in online supplemental figure 2A. There was no significant difference in respiratory viral load at admission (table 1). However, a high viral load in respiratory specimens after 1 week was found to be a risk factor for mortality (table 1 and online supplemental figure 2A). The median time (IQR) to positive conversion of immunofluorescent antibody (IFA) titre $(\geq 1: 640)$ for MERS-CoV from symptom onset was 18 days (17-23). Detailed kinetics of IFA titres in survivors and non-survivors are shown in online supplemental figure 2B. In addition, a low antibody titre at 2 weeks showed a trend towards association with mortality (low IFA antibody titre $(<1: 640)(100 \%$ (4/4, non-survivors)) compared with high antibody titre $(\geq 1: 640)(33 \% \quad(3 / 9$, survivors $)$, $\mathrm{p}=0.07$, table 1$)$. In summary, our data show that delayed viral clearance without an adequate antibody response 
Table 1 Baseline clinical characteristics, viral load, antibody titre, cytokine/chemokine and outcomes in 30 patients with Middle East respiratory syndrome (MERS)

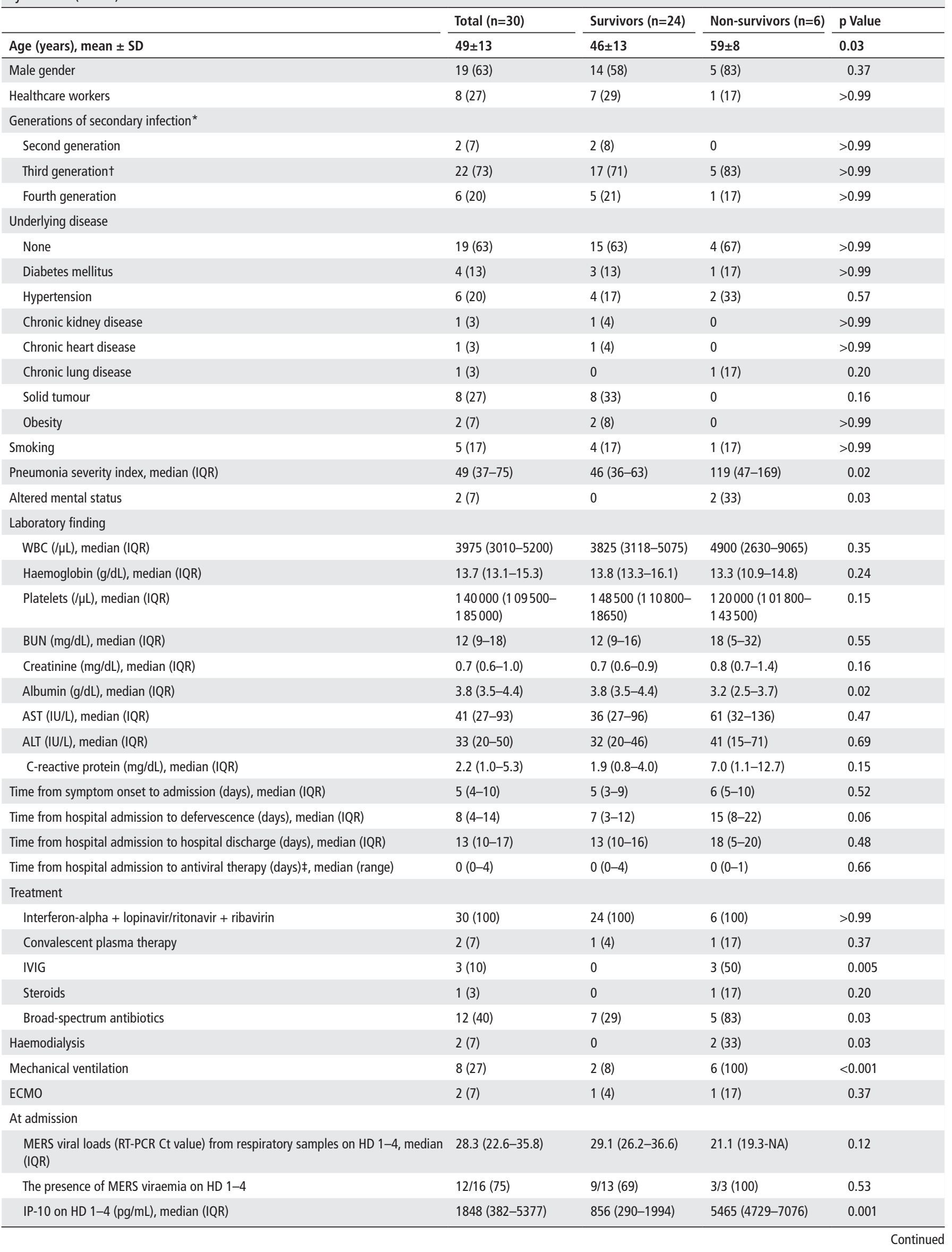




\begin{tabular}{|c|c|c|c|c|}
\hline & Total $(n=30)$ & Survivors $(n=24)$ & Non-survivors $(n=6)$ & p Value \\
\hline Age (years), mean \pm SD & $49 \pm 13$ & $46 \pm 13$ & $59 \pm 8$ & 0.03 \\
\hline MCP-1 on HD 1-4 (pg/mL), median (IQR) & $273(85-1498)$ & $93(54-324)$ & $1565(1171-2923)$ & $<0.001$ \\
\hline IL-6 on HD 1-4 (pg/mL), median (IQR) & $46(0-287)$ & $12(0-64)$ & $333(233-617)$ & $<0.001$ \\
\hline \multicolumn{5}{|l|}{ During the course of disease } \\
\hline $\begin{array}{l}\text { MERS viral loads (RT-PCR Ct value) of respiratory samples on HD 5-10 (1 week), } \\
\text { median (IQR) }\end{array}$ & $37.0(30.6-38.0)$ & $37.6(36.3-38.1)$ & 28.1 (23.7-NA) & $<0.001$ \\
\hline $\begin{array}{l}\text { MERS viral loads of (RT-PCR Ct value) respiratory samples on HD 11-16 } \\
\text { (2 weeks), median (IQR) }\end{array}$ & $36.4(33.9-38.5)$ & $36.8(34.6-38.7)$ & $29.0(31.8-\mathrm{NA})$ & 0.11 \\
\hline The presence of MERS viraemia on HD 5-10 (1 week) & $7 / 17(41)$ & $5 / 14(36)$ & $2 / 3(67)$ & 0.54 \\
\hline The presence of MERS viraemia on HD 11-16 (2 weeks) & $3 / 9(33)$ & $2 / 7(29)$ & $1 / 2(50)$ & $>0.99$ \\
\hline IP-10 on HD 5-10 (1 week) (pg/mL), median (IQR) & $840(298-2807)$ & $426(237-858)$ & $2991(1712-4230)$ & $<0.001$ \\
\hline MCP-1 on HD 5-10 (1 week) (pg/mL), median (IQR) & $179(80-526)$ & $124(46-219)$ & $532(276-656)$ & 0.005 \\
\hline IL-6 on HD 5-10 (1 week) (pg/mL), median (IQR) & $20(7-95)$ & $11(0-37)$ & $109(57-165)$ & 0.002 \\
\hline IP-10 on HD 11-16 (2 weeks) (pg/mL), median (IQR) & $419(249-851)$ & $253(171-314)$ & $791(441-1239)$ & $<0.001$ \\
\hline MCP-1 on HD 11-16 (2 weeks) (pg/mL), median (IQR) & $315(108-888)$ & $127(75-181)$ & $888(596-1183)$ & $<0.001$ \\
\hline IL-6 on HD 11-16 (2 weeks) (pg/mL), median (IQR) & $70(17-126)$ & $19(3-77)$ & $197(64-170)$ & 0.007 \\
\hline Low IFA antibody titre (<1:640) on HD 11-16 (2 weeks) & $7 / 13(54)$ & $3 / 9(33)$ & $4 / 4(100)$ & 0.07 \\
\hline $\begin{array}{l}\text { Low pseudovirus neutralisation assay antibody titre ( } \leq 1: 320 \text { ) on HD 17-24 } \\
\text { (3 weeks) }\end{array}$ & $9 / 12 \S(75)$ & $5 / 8(63)$ & $4 / 4(100)$ & 0.49 \\
\hline Low IFA antibody titre (<1:640) on HD 17-24 (3 weeks) & $4 / 6(67)$ & $1 / 3(33)$ & $3 / 3(100)$ & 0.40 \\
\hline Low pseudovirus neutralisation assay antibody titre ( $\leq 1: 320)$ on HD 17-24 & $3 / 6(50)$ & $1 / 3(33)$ & $2 / 3(67)$ & $>0.99$ \\
\hline
\end{tabular}

(3 weeks)

\begin{abstract}
*The first patient with confirmed MERS who triggered the 186 Korea MERS outbreak was defined as generation zero. The patients infected by this patient zero comprised the first generation, followed by the second generation, third generation and fourth generation.

tOne patient could not find any epidemiological link from this patient to the known confirmed MERS-CoV-infected patients in Korea outbreak, although exceptional epidemiological investigations were performed to determine the transmission chain by the Korean government. He was classified as third generation without high confidence. $¥$ Antiviral therapy includes interferon-alpha, ribavirin, or lopinavir/ritonavir.

$\S 0 f$ the 13 plasma samples, one sample was too small amount for the pseudovirus neutralisation assay and was not included.

ALT, alanine transaminase; AST, aspartate aminotransferase; BUN, blood urea nitrogen; CoV, coronavirus; ECMO, extracorporeal membrane oxygenation; HD 1-4, hospital days 1-4; IFA, immunofluorescent antibody; IL-6, interleukin-6; IP-10, inducible protein-10; IVIG intravenous immunoglobulin; MCP-1, monocyte chemoattractant protein-1; WBC, white blood cells.
\end{abstract}

during the late course of disease, rather than the initial viral load is associated with mortality (see online supplemental figure 2C).

\section{DISCUSSION}

It is well known that excessive expression of proinflammatory cytokines and chemokines released by activated immune cells and infected cells is also involved in immunopathology and the development of organ dysfunction. ${ }^{4}$ Indeed, the high concentrations of IP-10 and MCP-1 and the proinflammatory cytokine IL-6 in non-survivors with MERS indicate that the early excessive innate immune response is a critical factor leading to non-survival. IP-10 elevation during the early stage in non-survivors is reminiscent of its marked elevation in the early stage of severe acute respiratory syndrome (SARS). ${ }^{5}$ However, one study has reported that MCP-1 concentration is low during the early stage of SARS, ${ }^{5}$ although another study found that both IP-10 and MCP-1 were elevated in SARS. ${ }^{6}$ It is worth noting that the serum concentrations of IP-10, MCP-1 and IL-6 in non-survivors of MERS were highest in the early phase of MERS-CoV infection, suggesting that early intensive treatment is needed in patients with high serum concentrations of these factors to prevent organ dysfunction and reduce fatalities.

Interestingly, high viral loads during the late stage rather than the early stage of MERS were associated with mortality. In addition, inadequate antibody titres during the late stage showed a trend towards being associated with mortality. Old age has been reported to be an important risk factor for death in patients with MERS, ${ }^{7}$ which is consistent with our data (table 1). These demographic characteristics can be considered as proxies for the ability to amount an adequate immune response. Hence, poorly controlled virus replication due to an inadequate adaptive immune response, which is associated with host factors, is important in the pathogenesis of fatal MERS. In this context, more potent antiviral agents are urgently needed to improve the outcomes of MERS.

While the baseline characteristics and outcomes between 30 patients in this study and all the 186 patients in the Korea MERS outbreak were similar (data not shown), our findings may not be generalisable to other MERS outbreaks because neither the 186 patients nor the 30 patients enrolled in this study were representative of patients in the wide MERS outbreaks that occur in the Middle East. Finally, the number of patients $(n=30)$ was too small to perform multivariate analysis, so we could not rule out any potential confounding factors. In addition, this study might not have had sufficient power to detect the difference in characteristics between survivors 
A

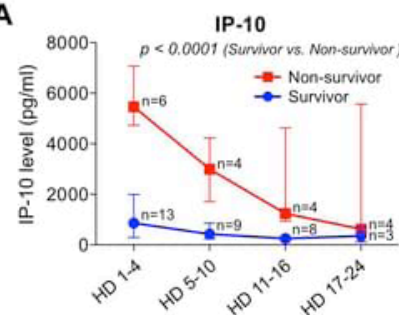

B

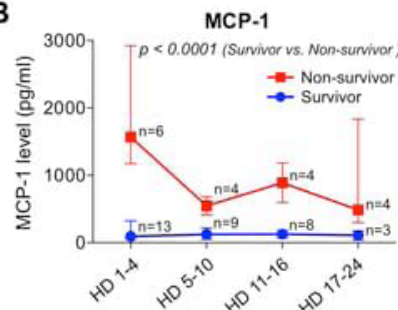

C

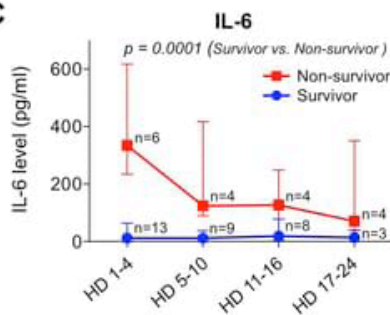

D

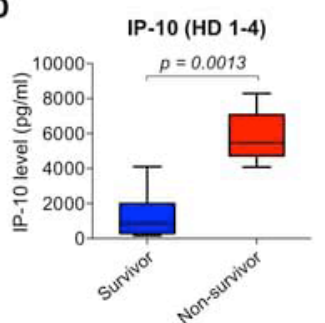

E
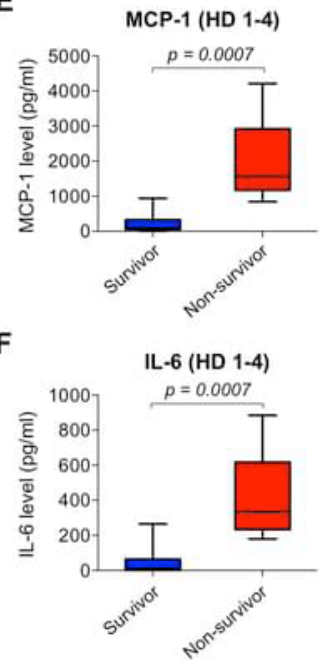

Contributors Conception and design: J-PC, HJC, E-CS, HP, S-HP, S-HK. Analysis and interpretation: K-HH, J-PC, E-CS, S-HP, S-HK. Data collection: K-HH, J-PC, S-HH, JL, J-SK, S-MK, SYP, J-YR, B-NK, S-HP, S-HK. Drafting the manuscript for important intellectual content: $\mathrm{K}-\mathrm{HH}$, E-CS, S-HP, S-HK

Funding This study was supported by grants from the Korea Health Technology R\&D Project through the Korea Health Industry Development Institute (KHIDI), funded by the Ministry of Health \& Welfare, Republic of Korea (HI15C2774, HI15C2859 and HI15C2888), from the National Research Foundation, funded by the Ministry of Science, ICT and Future Planning, Republic of Korea (NRF-2015R1A4A1042416), and from the Asan Institute for Life Sciences (Grant No. 2016462). It was also supported by the Korean Society for Chemotherapy.

Competing interests None declared.

Ethics approval Asan Medical Center Institutional Review Board.

Provenance and peer review Not commissioned; externally peer reviewed.

(C) Article author(s) (or their employer(s) unless otherwise stated in the text of the article) 2018. All rights reserved. No commercial use is permitted unless otherwise expressly granted.

- Additional material is published online only. To view please visit the journal online (http://dx.doi.org/ 10.1136/thoraxjnl-2016-209313).

\section{Check for updates}

Figure 1 (A-C) Serum inducible protein-10 (IP-10), monocyte chemoattractant protein-1 (MCP1) and IL-6 concentrations in patients with MERS. Serum concentrations of IP-10 (A), MCP-1 (B) and interleukin-6 (IL-6) (C) in recovering patients were significantly higher than those in nonsurvivors. The data are presented as medians with interquartile ranges. (D-F) Serum IP-10 (D) MCP-1 (E) and IL-6 (F) concentrations in HD 1-4 in survivors were significantly higher than those in non-survivors. The data are presented as box-and-whisker plots with the median, IQR and minimum to maximum values.

and non-survivors as shown in table 1. Therefore, our study results should be interpreted with caution.

In conclusion, severe inflammation at initial presentation, reflected in high IP-10, MCP-1 and IL-6 concentrations, and poor viral control without an adequate antibody response during the course of disease are associated with mortality in MERS-infected patients.

Ki-Ho Hong, ${ }^{1}$ Jae-Phil Choi, ${ }^{2}$ Seon-Hui Hong, ${ }^{3}$ Jeewon Lee, ${ }^{3}$ Ji-Soo Kwon, ${ }^{3,4}$ Sun-Mi Kim, ${ }^{4}$ Se Yoon Park, ${ }^{4}$ Ji-Young Rhee, ${ }^{5}$ Baek-Nam Kim, ${ }^{6}$ Hee Jung Choi, ${ }^{7}$ Eui-Cheol Shin, ${ }^{3,8}$ Hyunjoo Pai, ${ }^{9}$ Su-Hyung Park, ${ }^{3,8}$ Sung-Han Kim ${ }^{4}$

$\mathrm{K}-\mathrm{HH}, \mathrm{J}-\mathrm{PC}$ and S-HH contributed equally.

'Department of Laboratory Medicine, Seoul Medical Center, Seoul, Republic of Korea

${ }^{2}$ Department of Internal Medicine, Seoul Medical Center, Seoul, Republic of Korea

${ }^{3}$ Biomedical Science and Engineering Interdisciplinary Program, Korea Advanced Institute of Science and Technology, Daejeon, Republic of Korea
${ }^{4}$ Department of Infectious Diseases, Asan Medical Center, University of Ulsan College of Medicine, Seoul, Republic of Korea

5Division of Infectious Diseases, Department of Internal Medicine, Dankook University Hospital, Cheonan, Republic of Korea

${ }^{6}$ Department of Internal Medicine, Inje University Sanggye-Paik Hospital, Seoul, Rebulic of Korea

${ }^{7}$ Department of Internal Medicine, Ewha Womans University Hospital, Seoul, Republic of Korea

${ }^{8}$ Graduate School of Medical Science and Engineering, Korea Advanced Institute of Science and Technology, Daejeon, Republic of Korea

${ }^{9}$ Department of Internal Medicine, Han Yang University Hospital, Seoul, Republic of Korea

Correspondence to Dr Su-Hyung Park, Laboratory of Translational Immunology and Vaccinology, Graduate School of Medical Science and Engineering, KAIST, Daejeon, 34141, Republic of Korea; park3@kaist.ac. $\mathrm{kr}$ and Dr Sung-Han Kim, Department of Infectious Diseases, Asan Medical Center, University of Ulsan College of Medicine, 388-1 Pungnap-dong, Songpa-gu, Seoul, 05505, Republic of Korea; kimsunghanmd@ hotmail.com

Acknowledgements We thank Dr Ho-Sung Seo (Korea Atomic Energy Research Institute) for ${ }^{60} \mathrm{Co}$ gamma irradiation.
To cite Hong K-H, Choi J-P, Hong S-H, et al. Thorax 2018:73:286-289.

Received 15 August 2016

Revised 10 May 2017

Accepted 15 May 2017

Published Online First 19 July 2017

Thorax 2018;73:286-289.

doi:10.1136/thoraxinl-2016-209313

\section{REFERENCES}

1 Faure E, Poissy J, Goffard A, et al. Distinct immune response in two MERS-CoV-infected patients: can we go from bench to bedside? PLoS One 2014:9:e88716.

2 Min CK, Cheon S, Ha NY, et al. Comparative and kinetic analysis of viral shedding and immunological responses in MERS patients representing a broad spectrum of disease severity. Sci Rep 2016:6:25359.

3 Kumar M, Mazur S, Ork BL, et al. Inactivation and safety testing of Middle East respiratory syndrome coronavirus. J Virol Methods 2015;223:13-18.

4 Huang KJ, Su IJ, Theron M, et al. An interferon-gammarelated cytokine storm in SARS patients. J Med Virol 2005;75:185-94.

5 Jiang Y, Xu J, Zhou C, et al. Characterization of cytokine/chemokine profiles of severe acute respiratory syndrome. Am J Respir Crit Care Med 2005:171:850-7.

6 Wong CK, Lam CW, Wu AK, et al. Plasma inflammatory cytokines and chemokines in severe acute respiratory syndrome. Clin Exp Immunol 2004;136:95-103.

7 Hui DS, Memish ZA, Zumla A. Severe acute respiratory syndrome vs. the Middle East respiratory syndrome. Curr Opin Pulm Med 2014:20:233-41. 